Society of Pacing and Electrophysiology, adapted for preterm infants. These features were mean NN (ms), SDNN (ms), mean VLF (ms2), mean LF (ms2), mean HF (ms2), LF/HF ratio and TINN (ms).

These HRV features were then individually tested for group differences among the outcome groups using the appropriate Mann-Whitney U test.

Results Nine of the twenty-eight babies developed IVH.

4 infants had Grade 1 and 5 infants had Grade 2 IVH.

None of the infants had a severe IVH (grade 3/4).

Overall there was no difference in all features between the two individual time points for the group overall.

There were no statistically significant differences in the features between infants with and without low grades of IVH.

Conclusions We found no association between HRV characteristics at 6 and 12 hours of age and low grades of IVH in preterm infants less than 32 weeks. Future work is needed to explore the relationship between HRV and severe IVH (grade 3/4).

\section{GP252 THE EFFECT OF MUSIC THERAPY ON THE ELECTROENCEPHALOGRAM (EEG) AND HEART RATE VARIABILITY (HRV) OF PREMATURE INFANTS DURING ROUTINE PAINFUL PROCEDURES}

\begin{abstract}
${ }^{1,2}$ Andreea M Pavel*, ${ }^{1}$ Farah Abu Dhais, ${ }^{2}$ Caoimhe Howard, ${ }^{1}$ John M O'Toole, ${ }^{1}$ Elena Pavlidis, ${ }^{1,3}$ Daragh Finn, ${ }^{1}$ Vicki Livingstone, ${ }^{4}$ Anna Powell, ${ }^{1,2}$ Eugene M Dempsey, ${ }^{1}$ Geraldine B Boylan. ${ }^{1}$ INFANT Centre, University College Cork, Cork, Ireland; ' ${ }^{2}$ Cork University Maternity Hospital, Cork, Ireland; ${ }^{3}$ Cork University Hospital, Cork, Ireland; ${ }^{4}$ University College Cork, Cork, Ireland
\end{abstract}

\subsection{6/archdischild-2019-epa.311}

Background Music therapy may be beneficial for relieving pain associated with Neonatal Intensive Care Unit procedures. The aim of this study was to assess the effect of music on the EEG and HRV of premature infants during painful procedures.

Methods This was a randomised crossover study in newborns delivered before 32 weeks gestational age (GA). Infants were randomised to receive initially either sucrose or sucrose and music therapy (Brahms' lullaby) during routine venepuncture. Infants had multichannel EEG recorded during the procedure, with ECG part of the EEG montage. Three 2-minute artefact free EEG and ECG epochs (before venepuncture, 4 minutes and 18 minutes after venepuncture) were selected for analysis. Matlab was used to perform quantitative EEG and HRV analysis.

Results

Twenty-one preterm infants were recruited 8 males (38\%) and 13 females (62\%), mean birth weight 932 g. The EEG analysis showed no clinically significant difference between the two groups. The HRV analysis showed an increase of the mean NN interval and LF-HF ratio at 18 minutes post-procedure in intervention arm.

Conclusion EEG features were not significantly altered by painful procedures in our study, and music therapy did not change this response. The changes in HRV indicate improved autonomic stability and a decrease stress during painful procedures with music therapy.

\section{GP253 MATERNAL AWARENESS, ACCEPTABILITY AND WILLINGNESS TOWARDS RESPIRATORY SYNCYTIAL VIRUS (RSV) VACCINATION DURING PREGNANCY}

${ }^{1}$ Siobhan McCormack*, 'Claire Thompson, ${ }^{1}$ Caitriona Ní Chathasaigh, ${ }^{2}$ Miriam Nolan, ${ }^{2}$ Mendinaro Imcha, ${ }^{3}$ Ann Dee, ${ }^{4}$ Jean Saunders, ${ }^{1,5}$ Roy K Philip. ${ }^{1}$ Division of Neonatology, Department of Paediatrics, University Maternity Hospital Limerick, Limerick, Ireland; ${ }^{2}$ Department of Obstetrics and Gynaecology, University Maternity Hospital, Limerick, Ireland; ${ }^{3}$ Department of Public Health, Health Service Executive, Limerick, Ireland; ${ }^{4}$ Statistical Consulting Unit, University of Limerick, Limerick, Ireland; ${ }^{5}$ Graduate Entry Medical School (GEMS), University of Limerick, Limerick, Ireland

\subsection{6/archdischild-2019-epa.312}

Background RSV is the leading cause of viral lower respiratory tract infections and hospital admissions among infants. There were over 3,100 cases of RSV reported in Ireland in winter $2017 / 2018$ and globally, RSV is responsible for over 1.4 million admissions and 27,000 deaths in Infants under 6 months annually. A vaccine against RSV has been identified as a priority by the World Health Organisation (WHO) and several candidate vaccines against RSV are currently in clinical development.

Aims To evaluate maternal knowledge of RSV infection and to assess likely acceptance if an approved licenced vaccine for use in pregnancy is made available in Ireland.

Methods Pregnant women were surveyed prospectively during normal and high-risk antenatal clinic visits. Information leaflet was provided, consent for the survey obtained and ethical approval was granted by the UL Hospitals Group research ethics committee. Study design proposed 900 pregnant women for consecutive enrolment.

Results The response from the initial part of the survey was analysed. Mean age of women surveyed was 32 years (range $17-42$ years) while gestation ranged from 11 to 40 weeks of gestation (mean 29 weeks). 57\% of women were in their first pregnancy, $20 \%$ in their second and the remaining $23 \%$ in their third or subsequent pregnancies.

$71 \%$ of women had never heard of RSV, 19\% had some knowledge of it, $10 \%$ had knowledge of its significance in infancy and interestingly no respondents among the initial cohort analysed had experience of it in their own child. 57\% of women responded that if an antenatal vaccine against RSV was available, they would accept it and $37 \%$ of respondents were unsure.

$35 \%$ of women felt that recommended vaccines would protect their infant from illness, $21 \%$ were confident in recommended vaccines, and a further $29 \%$ believed that vaccines would both protect their infant from illness and felt confident in the potential recommended vaccine. A combined positive response was noted from $85 \%$ of pregnant women.

54\% indicated consulting their GP best influencing their decision making, 23\% preferred midwife, 14\% opted for discussion with obstetrician, 4\% information leaflets, 3\% depended on a relative and 1\% online resources.

Conclusion Over half of women indicated that they would accept a vaccine to protect their infants against with high rates of vaccine confidence. Women's preferences for advice regarding vaccination during pregnancy should be considered when planning such programmes given the strong desire for information seeking from primary care setting. 